\title{
Fatores de risco cardiovascular em universitários: comparação entre se- xos, períodos de graduação e áreas de estudo
}

\section{Cardiovascular risk factors in college students: comparison among sexes, undergraduation period and study areas}

\author{
Guilherme da S. Gasparotto', Lívia P. R. Gasparotto², Maicon R. de Salles¹, Wagner de Campos ${ }^{1,3}$
}

\begin{abstract}
RESUMO
Modelo do estudo: Estudo de corte transversal comparativo. Objetivo: Realizar comparações dos fatores de risco entre sexos, estudantes calouros e formandos e entre áreas de estudo. Metodologia: Os comportamentos de prática de atividade física, tabagismo, etilismo, hábitos alimentares foram obtidos por método recordatório através dos questionários IPAQ e YRBSS, foram realizadas medidas de peso e estatura para o IMC e ainda circunferência de cintura e pressão arterial. Resultados: Todas variáveis contínuas foram superiores em homens comparados às mulheres $(p<0,01)$. A circunferência de cintura e pressão arterial sistólica foram superiores em formandos do que em calouros $(p<0,01)$. A circunferência de cintura, pressão arterial sistólica e diastólica superiores em alunos de ciências Exatas frentes às outras áreas. Apresentaram-se como Insuficientemente Ativos 54,6\% dos alunos, $64,8 \%$ consumiram álcool no último mês e $92,1 \%$ não cumpriram a recomendação de três ou mais porções de frutas por dia. Conclusão: Foi possível observar importantes diferenças nos fatores de risco relacionados à atividade física moderado-vigorosa, tabagismo, consumo de álcool, IMC, circunferência de cintura e hábitos alimentares entre os grupos, o que demonstra necessidade de investimentos para mudanças em comportamentos entre os estudantes.
\end{abstract}

Palavras chave: Fatores de Risco. Estilo de Vida. Estudantes. Obesidade. Atividade Física.

\section{Introdução}

Devido à alta taxa de mortes atribuída às doenças cardiovasculares (DCVs), diversas pesquisas são direcionadas aos fatores de risco para o desenvolvimento deste tipo de patologia. ${ }^{1,2,3}$ As Diretrizes de
Prevenção contra Doenças Cardiovasculares ${ }^{4}$ enfatizam a necessidade de cuidados relativos a comportamentos como sedentarismo, consumo de álcool e tabaco, hábitos alimentares, condição de excesso de peso corporal e pressão arterial elevada. A importância destes fatores deve-se à sua influência no desen-
1- Pesquisador do Centro de Estudos em Exercício e Esporte (CEEPE) - Universidade Federal do Paraná

2- Professora do curso de Saúde Coletiva da Universidade Federal do Paraná - Setor Litoral

3- Professor orientador do programa de Pós Graduação em Educação Física da Universidade Federal do Paraná
Correspondência: Guilherme da S. Gasparotto Departamento de Educação Física Rua Coração de Maria, 92. Campus Jardim Botânico CEP 80210-132 - Curitiba/Paraná Email: guilhermegptt@gmail.com

Artigo recebido em 03/07/2012 Aprovado para publicação em 25/06/2013 
volvimento de doenças como infarto agudo do miocárdio, insuficiência cardíaca, diabetes, acidente vascular encefálico (AVE), entre outras.

Atualmente, relevante parte das investigações sobre comportamentos e fatores metabólicos de risco à saúde é direcionada à população de jovens adultos 1,5,6. Pois é sabido que fatores de risco adquiridos durante esta fase podem perdurar durante o envelhecimento e auxiliar no desenvolvimento de DCVs. ${ }^{7,8}$

A atenção para esta faixa etária refere-se ao fato do não aparecimento dos sintomas nesta fase, ainda que o organismo já apresente irregularidades fisiológicas. Pesquisadores apontam que a falta de sintomas agudos, aliada ao longo prazo do desenvolvimento das doenças cardiovasculares auxiliam que estes jovens adultos apresentem-se mais displicentes quanto aos cuidados com os fatores de risco. ${ }^{9,10}$

Neste contexto, o período universitário pode ser visto como importante fase na vida do indivíduo, pois o novo meio social em qual o estudante é inserido parece influenciar no seu estilo de vida habitual, gerando modificações relevantes quanto aos comportamentos. Desta forma o aluno estaria mais suscetível a aquisição de fatores de risco para o desenvolvimento de DCVs. ${ }^{11,10}$ A presença destes fatores parece ainda ser diferente em grupos específicos dentro desta população, como entre sexos, alunos calouros e formandos ou entre diferentes áreas de estudo. ${ }^{12,13}$

Verifica-se com frequência a comparação da presença de fatores de risco entre os sexos em estudos com universitários, no entanto a comparação destes fatores entre períodos de graduação e áreas de estudo foram pouco exploradas. Além disto, estudos com esta população geralmente são elaborados com amostras intencionais, não probabilísticas, o que acaba dificultando conclusões acerca das comparações entre os grupos de universitários estudados. ${ }^{6,12,13}$ Neste contexto, o propósito do presente estudo foi comparar a presença de fatores de risco cardiovascular entre sexos, estudantes calouros e formandos e diferentes áreas de estudo, bem como a prevalência destes de acordo com os pontos de corte estabelecidos para cada fator.

\section{Métodos}

\section{População e amostra}

Este estudo foi realizado com amostra representativa, proveniente de alunos da Universidade Fe- deral do Paraná (UFPR), que apresentou aproximadamente 24.000 estudantes matriculados em diversos cursos de todas as áreas conhecimento. A população alvo da pesquisa foi composta por alunos de primeiro (5.059) e último (1.582) ano da graduação totalizando 6.641 indivíduos.

A definição da amostra foi realizada utilizando a calculadora Epiinfo, desenvolvida pelo Center of Desease Control Prevention (CDC). Para a realização do cálculo se levou em consideração: Nível de confiança de $95 \%$ e Poder amostral de $80 \%$. Utilizouse também os valores da variável IMC apresentados por Ribeiro e Fernandes ${ }^{14}$ para indivíduos expostos e não expostos ao baixo nível de atividade física, onde foi utilizado método, instrumento de avaliação e amostra similares à do presente estudo. A amostra mínima calculada foi de 986 e com a correção de desenho amostral (deff) de 1,515, devido à estratificação por conglomerados, mais $10 \%$ para possíveis perdas de dados, a amostra final foi estimada em 1.577 indivíduos. Após definição do número amostral, este foi dividido proporcionalmente em áreas de estudo (Ciências Humanas, Exatas ou Biológicas), ingressantes e formandos e turno de estudo (Diurno e Noturno).

\section{Instrumentos e Procedimentos}

Para a estimativa do nível de atividade física (NAF) foi utilizado o International Physical Activity Questionaire (IPAQ), levando em consideração o tempo acumulado de atividade física moderada e vigorosa durante a última semana. $\mathrm{O}$ ponto de corte adotado foi de 150 minutos ou mais, como sugere o Global Recommendations on Physical Activity for Health. ${ }^{16}$

Os comportamentos de consumo de álcool, tabaco e hábitos alimentares foram avaliados mediante o YRBS-C (Youth Risk Behavior Surveillance) desenvolvido pelo CDC e previamente validado para universitários brasileiros. ${ }^{17}$ Os estudantes foram questionados sobre quantas vezes nos trinta dias anteriores à pesquisa fizeram uso de álcool e tabaco e quantas vezes neste mesmo período consumiram cinco doses ou mais de bebida alcoólica. Foram ainda coletadas informações sobre a quantidade de porções consumidas no dia anterior de frutas, saladas ou vegetais cozidos, salgados (batata frita, salgadinhos ou similares) e doces (bolos, biscoitos e similares). Foi adotado ponto de corte três ou mais porções para o consumo de frutas e quantidade igual para saladas e/ou vegetais, como sugerido pelo Dietary Guidelines for Americans. ${ }^{2}$ 
Os indicadores antropométricos peso e estatura para cálculo do IMC e Circunferência da Cintura (CC) foram utilizados para definição de sobrepeso ou obesidade. Os pontos de corte adotado para esses índices seguiram os valores sugeridos pela Organização Mundial da Saúde, sendo o IMC em $25 \mathrm{Kg} / \mathrm{m}^{2}$ e para CC $94 \mathrm{~cm}$ em homens e $80 \mathrm{~cm}$ em mulheres. ${ }^{4}$

A pressão arterial (PA) foi verificada através do método auscultatório de Karotkoff utilizando um esfigmomanômetro aneróide e estetoscópio, com ponto de corte de $140 \mathrm{mmHg}$ para pressão sistólica e 90 mmHg para diastólica como sugerido pela OMS. ${ }^{4}$

A coleta de dados ocorreu entre os meses de março e julho de 2011 durante o período de aula de cada turma, seguindo a sequência: explicação dos procedimentos e assinatura do termo de consentimento livre e esclarecido (TCLE), verificação da pressão arterial, preenchimento dos questionários e medidas antropométricas de peso, estatura e circunferência da cintura.

O estudo foi aprovado pelo Comitê de Ética em Pesquisa com seres humanos do setor de Ciências da Saúde da Universidade Federal do Paraná, estando de acordo com a resolução n. 196/1996 do Conselho Nacional de Saúde, sob o registro CEP/SD: 1043.168.10.11.

\section{Análise estatística}

A verificação da normalidade dos dados foi feita a partir do teste de Kolgomorov-Smirnov. A comparação das variáveis contínuas, tempo em AFMV, IMC, CC, PAS e PAD entre sexos e período de graduação foi analisada pelo teste t-Student e entre as três áreas de estudo através da análise de variância (ANOVA) e posteriormente aplicação do Post Hoc de Tukey. Distribuições de frequência foram realizadas para análise de prevalências das variáveis: cumprimento da recomendação de AFMV, comportamentos de etilismo, tabagismo, hábitos alimentares, IMC elevado, CC elevada e pressão arterial elevada. A comparação das prevalências destas variáveis foi analisada através do teste de Qui-Quadrado. A significância foi estipulada em $p<0,05$. Todas as análises foram realizadas através do pacote estatístico SPSS 18.0.

\section{Resultados}

Entre os 6.641 universitários elegíveis, foram avaliados 1.599, sendo 1.197 ingressantes e 402 formandos. A média de idade da amostra total foi de $20,8 \pm 3,9$ anos.

A análise comparativa entre sexos, da média e desvios padrão das variáveis contínuas, mostrou que os homens apresentaram maior tempo gasto em AFMV, IMC, CC, PAS e PAD do que as mulheres, como expõe a tabela 1.

A análise comparativa entre períodos de graduação, da média e desvios padrão das variáveis contínuas, mostrou que houve superioridade entre os formandos para CC e para calouros em tempo gasto com AFMV, como demonstra a tabela 2.

A análise comparativa entre áreas de estudo, da média e desvios padrão das variáveis contínuas, mostrou maior tempo gasto em AFMV entre estudantes de ciências Biológicas, comparado aos de ciências Exatas e Humanas. Já o IMC, CC, PAS e PAD foram superiores entre os estudantes de ciências Exatas, como expõe a tabela 3 abaixo.

\section{Tabela 1}

Comparação das médias dos fatores de risco cardiovascular entre sexos

\begin{tabular}{|c|c|c|c|c|c|c|c|c|}
\hline \multirow{2}{*}{$\frac{\text { Variáveis }}{\text { Idade (anos) }}$} & \multicolumn{2}{|c|}{$\begin{array}{c}\text { Geral } \\
\text { Média(DP) }\end{array}$} & \multicolumn{3}{|c|}{$\begin{array}{l}\text { Masculino } \\
\text { Média(DP) }\end{array}$} & \multicolumn{2}{|c|}{$\begin{array}{c}\text { Feminino } \\
\text { Média (DP) }\end{array}$} & \multirow{2}{*}{$\frac{\mathbf{p}}{0,51}$} \\
\hline & $20,7 \pm$ & 3,9 & 20,8 & \pm & 4,1 & $20,7 \pm$ & 3,8 & \\
\hline AFMV min./semana & $89,3 \pm$ & 107,7 & 94,8 & \pm & 99,5 & $84,1 \pm$ & 114,9 & $0,04 *$ \\
\hline $\operatorname{IMC}\left(\mathrm{kg} / \mathrm{m}^{2}\right)$ & $23,3 \pm$ & 4 & 23,8 & \pm & 4,2 & $22,7 \pm$ & 3,7 & $<0,01 *$ \\
\hline Circunferência da cintura $(\mathrm{cm})$ & $75,3 \pm$ & 9,8 & 79,8 & \pm & 9,7 & 71 & 7,7 & $<0,01 *$ \\
\hline Pressão arterial sistólica (mmHg) & $113,9 \pm$ & 12,9 & 118 & \pm & 12,3 & $109,4 \pm$ & 11,9 & $<0,01^{*}$ \\
\hline Pressão arterial diastólica (mmHg) & $75,2 \pm$ & 9,6 & 78 & \pm & 9,5 & $72,5 \pm$ & 9 & $<0,01 *$ \\
\hline
\end{tabular}

AFMV: Atividade Física Moderado-Vigorosa; IMC: Índice de Massa Corporal; DP: Desvio Padrão; $\mathrm{p}^{*}<0,05$ 


\section{Tabela 2}

Comparação das médias dos fatores de risco cardiovascular entre períodos de graduação

\begin{tabular}{|c|c|c|c|c|}
\hline Variáveis & $\begin{array}{c}\text { Geral } \\
\text { Média(DP) }\end{array}$ & $\begin{array}{c}\text { Calouros } \\
\text { Média(DP) }\end{array}$ & $\begin{array}{l}\text { Formandos } \\
\text { Média (DP) }\end{array}$ & $\mathbf{p}$ \\
\hline Idade (anos) & $20,7 \pm$ & $20,1 \pm 3,8$ & $22,9 \pm$ & $<0,01^{*}$ \\
\hline AFMV min./semana & $89,3 \pm 107,7$ & $92,2 \pm 106,1$ & $80,8 \pm 112,0$ & $0,04 *$ \\
\hline $\operatorname{IMC}\left(\mathrm{kg} / \mathrm{m}^{2}\right)$ & $23,3 \pm 4,0$ & $23,2 \pm 4,0$ & $23,5 \pm$ & 0,11 \\
\hline Circunferência da cintura $(\mathrm{cm})$ & $75,3 \pm$ & $\pm \quad 9,9$ & $76,3 \pm$ & $0,01^{*}$ \\
\hline Pressão arterial sistólica (mmHg) & $113,9 \pm 12,9$ & $113,1 \pm 12,9$ & $116,1 \pm 12,7$ & $<0,01^{*}$ \\
\hline Pressão arterial diastólica (mmHg) & $75,2 \pm$ & $74,9 \pm 9,6$ & $75,9 \pm$ & 0,08 \\
\hline
\end{tabular}

AFMV: Atividade Física Moderado-Vigorosa; IMC: Índice de Massa Corporal; DP: Desvio Padrão; $p^{*}<0,05$

Tabela 3

Comparação das médias dos fatores de risco cardiovascular entre áreas de estudo

\begin{tabular}{|c|c|c|c|c|c|}
\hline Variáveis & $\begin{array}{c}\text { Geral } \\
\text { Média(DP) }\end{array}$ & $\begin{array}{c}\text { Exatas } \\
\text { Média (DP) }\end{array}$ & $\begin{array}{c}\text { Humanas } \\
\text { Média(DP) }\end{array}$ & $\begin{array}{l}\text { Biológicas } \\
\text { Média(DP) }\end{array}$ & $\mathbf{P}$ \\
\hline Idade & $20,7 \pm$ & $21,2 \pm 4,2^{\mathrm{a}-\mathrm{b}}$ & $20,5 \pm$ & $20,5 \pm$ & $<0,01^{*}$ \\
\hline AFMV min./semana & $89,3 \pm 107,7$ & $88,6 \pm 92,4$ & $79,5 \pm 108,1$ & $107,0 \pm 124,1^{\mathrm{a}-\mathrm{c}}$ & $<0,01^{*}$ \\
\hline $\mathrm{IMC}\left(\mathrm{kg} / \mathrm{m}^{2}\right)$ & $23,3 \pm 4,0$ & $23,6 \pm 4,0$ & $23,1 \pm$ & $23,0 \pm 3,9$ & 0,63 \\
\hline Circunferência da cintura $(\mathrm{cm})$ & $75,3 \pm 9,8$ & $77,6 \pm 10,0^{\mathrm{a}-\mathrm{b}}$ & $74,2 \pm$ & $73,8 \pm$ & $<0,01^{*}$ \\
\hline Pressão arterial sistólica (mmHg) & $113,9 \pm 12,9$ & $116,5 \pm 12,3^{a-b}$ & $113,0 \pm 12,7$ & $111,0 \pm 13,5$ & $<0,01 *$ \\
\hline Pressão arterial diastólica ( $\mathrm{mmHg}$ ) & $75,2 \pm$ & $76,2 \pm 9,2^{b}$ & $75,2 \pm 10,1$ & $74,0 \pm$ & $<0,01 *$ \\
\hline
\end{tabular}

AFMV: Atividade Física Moderado-Vigorosa; IMC: Índice de Massa Corporal; DP: Desvio Padrão; $a=$ maior que em Humanas, b = maior que em Biológicas, $\mathrm{c}=$ maior que Exatas, $\mathrm{p}^{*}<0,05$

Entre sexos, as mulheres apresentaram menor prevalência no cumprimento da recomendação de AFMV e maior prevalência de consumo de doces no dia anterior à pesquisa. Já os homens tiveram superioridade nas prevalências de utilização de tabaco nos últimos trinta dias, consumo de bebida alcoólica, bem como seu consumo em excesso também nos últimos trinta dias, consumo insuficiente de frutas e saladas e/ ou vegetais, IMC elevado e pressão arterial elevada, como apresenta a tabela 4.

Formandos apresentaram maiores prevalências para prática insuficiente de AFMV, utilização de taba- co nos últimos trinta dias, consumo de álcool e seu consumo em excesso em trinta dias, como apresenta a tabela 5.

Verificou-se superioridade nas prevalências de alunos das ciências Exatas nos seguintes fatores: consumo de álcool e de mais de cinco doses numa única ocasião em trinta dias e IMC elevado. Os alunos de ciências Humanas apresentaram maiores prevalências de prática insuficiente de AFMV. Já os alunos dos cursos de ciências Biológicas apresentaram maior prevalência para o consumo de doces no dia anterior à pesquisa, como mostra a tabela 6 . 


\section{Tabela 4}

Comparação das prevalências dos fatores de risco cardiovascular entre sexos

\begin{tabular}{|c|c|c|c|c|c|c|c|}
\hline \multirow[b]{2}{*}{ Variáveis } & \multicolumn{2}{|c|}{ Geral } & \multicolumn{2}{|c|}{ Masculino } & \multicolumn{2}{|c|}{ Feminino } & \multirow[b]{2}{*}{$\mathbf{p}$} \\
\hline & $\% \mathrm{~N}$ & IC - 95\% & $\%$ & IC - 95\% & $\% \mathrm{~N}$ & IC - $95 \%$ & \\
\hline Idade $>20$ & $53,0(847)$ & $50,5-55,2$ & $54,6(443)$ & $51,1-58,3$ & $51,3(404)$ & $48,0-55,1$ & 0,09 \\
\hline AFMV $<150$ min./semana & $54,6(871)$ & $51,9-57,1$ & $48,7 \%(384)$ & $45,4-52,3$ & $60,2 \%(488)$ & $56,6-63,6$ & $<0,01^{*}$ \\
\hline Uso de tabaco em 30 dias & $11,4(180)$ & $9,7-12,8$ & $14,4 \%(113)$ & $6,4-10,2$ & $8,3 \%(67)$ & $6,4-10,2$ & $<0,01 *$ \\
\hline Uso de álcool em 30 dias & $64,8(1033)$ & $62,2-67,3$ & $69 \%(542)$ & $65,9-72,4$ & $60,0 \%(491)$ & $57,2-63,8$ & $<0,01^{*}$ \\
\hline Excesso de álcool em 30 dias & $40,2(639)$ & $37,6-42,5$ & $47,5 \%(373)$ & $44,3-51,0$ & $32,8 \%(266)$ & $29,5-35,9$ & $<0,01^{*}$ \\
\hline $\begin{array}{l}\text { Frutas }<3 \text { porções no } \\
\text { dia anterior }\end{array}$ & $92,1(1470)$ & $90,7-93,4$ & $92,2 \%(745)$ & $90,3-94,0$ & $92,0 \%(725)$ & $90,0-93,7$ & 0,92 \\
\hline $\begin{array}{l}\text { Saladas/vegetais }<3 \text { porções } \\
\text { no dia anterior }\end{array}$ & $80,3(1281)$ & $78,3-82,3$ & $82,8 \%(651)$ & $80,0-85,2$ & $77,8 \%(630)$ & $74,9-80,7$ & $0,01 *$ \\
\hline $\begin{array}{l}\text { Consumiu salgado } \\
\text { no dia anterior }\end{array}$ & $34,7(553)$ & $32,6-37,1$ & $33,2 \%(261)$ & $30,1-36,4$ & $36,0 \%(292)$ & $32,6-39,3$ & 0,24 \\
\hline $\begin{array}{l}\text { Consumiu doce } \\
\text { no dia anterior }\end{array}$ & $68,9(1099)$ & $66,8-71,2$ & $66,1 \%(519)$ & $62,7-69,3$ & $71,6 \%(580)$ & $68,5-74,6$ & $0,02 *$ \\
\hline $\mathrm{IMC}>25 \mathrm{~kg} / \mathrm{m}^{2}$ & $25,9(413)$ & $23,7-28$ & $32 \%(251)$ & $28,8-35,0$ & $20,0 \%(162)$ & $17,3-22,8$ & $<0,01 *$ \\
\hline $\begin{array}{l}C C \geq 80 \text { feminino e } \\
\geq 94 \text { masculino }(\mathrm{cm})\end{array}$ & $9,9(158)$ & $8,5-11,3$ & $10,4 \%(84)$ & $7,4-11,5$ & $9,4 \%(74)$ & $8,4-12,6$ & 0,55 \\
\hline $\mathrm{PA}>140 / 90(\mathrm{mmHg})$ & $9,5(152)$ & $8,1-11,0$ & $14,3 \%(112)$ & $11,7-16,2$ & $4,9 \%(40)$ & $3,5-6,4$ & $<0,01 *$ \\
\hline
\end{tabular}

AFMV: Atividade Física Moderado-Vigorosa; IMC: Índice de Massa Corporal; CC: Circunferência da Cintura; DP: Desvio Padrão; PA: Pressão Arterial; IC: Intervalo de Confiança; $\mathrm{p}^{*}<0,05$

\section{Tabela 5}

Comparação das prevalências dos fatores de risco cardiovascular entre períodos de graduação

\begin{tabular}{|c|c|c|c|c|c|c|c|}
\hline \multirow[b]{2}{*}{ Variável } & \multicolumn{2}{|c|}{ Geral } & \multicolumn{2}{|c|}{ Calouros } & \multicolumn{2}{|c|}{ Formandos } & \multirow[b]{2}{*}{$\mathbf{p}$} \\
\hline & $\% \mathbf{N}$ & IC - $95 \%$ & $\% \mathbf{N}$ & IC $-95 \%$ & $\% \mathrm{~N}$ & IC $-95 \%$ & \\
\hline Idade $>21$ & $53(847)$ & $50,5-55,2$ & $37,5(449)$ & $34,9-40,4$ & $99(398)$ & $98-99,8$ & $<0,01^{*}$ \\
\hline AFMV $<150$ min./semana & $54,6(871)$ & $51,9-57,1$ & $52,8 \%(632)$ & $49,7-55,6$ & $59,7 \%(240)$ & $54,5-64,2$ & $0,02 *$ \\
\hline Uso de tabaco em 30 dias & $11,4(180)$ & $9,7-12,8$ & $9,6 \%(115)$ & $8-11,3$ & $16,2 \%(65)$ & $12,4-19,9$ & $<0,01^{*}$ \\
\hline Uso de álcool em 30 dias & $64,8(1033)$ & $62,2-67,3$ & $60,9 \%(726)$ & $57,9-63,5$ & $76,4 \%(307)$ & $72,1-80,6$ & $<0,01 *$ \\
\hline Excesso de álcool em 30 dias & $40,2(639)$ & $37,6-42,5$ & $35,2 \%(420)$ & $32,9-38$ & $54,5 \%(219)$ & $49,8-59,5$ & $<0,01 *$ \\
\hline $\begin{array}{l}\text { Frutas }<3 \text { porções no } \\
\text { dia anterior }\end{array}$ & $92,1(1470)$ & $90,7-93,4$ & $92,4 \%(1103)$ & $91-93,8$ & $91,3 \%(367)$ & $88,6-93,8$ & 0,52 \\
\hline $\begin{array}{l}\text { Saladas/vegetais }<3 \text { porções } \\
\text { no dia anterior }\end{array}$ & $80,3(1281)$ & $78,3-82,3$ & $79,4 \%(948)$ & $77,1-81,7$ & $82,9 \%(333)$ & $79,1-86,3$ & 0,14 \\
\hline $\begin{array}{l}\text { Consumiu salgado no } \\
\text { dia anterior }\end{array}$ & $34,7(553)$ & $32,6-37,1$ & $34,5 \%(412)$ & $31,9-37,4$ & $35,1 \%(141)$ & $30,6-39,6$ & 0,85 \\
\hline $\begin{array}{l}\text { Consumiu doce no } \\
\text { dia anterior }\end{array}$ & $68,9(1099)$ & $66,8-71,2$ & $69,9 \%(834)$ & $67,5-72,5$ & $65,9 \%(265)$ & $60,9-70,4$ & 0,15 \\
\hline $\mathrm{IMC} \geq 25 \mathrm{~kg} / \mathrm{m}^{2}$ & $25,9(413)$ & $23,7-28$ & $24,7 \%(295)$ & $22,4-27,3$ & $29,4 \%(118)$ & $24,9-33,8$ & 0,06 \\
\hline $\begin{array}{l}C C \geq 80 \text { feminino e } \\
>94 \text { masculino }(\mathrm{cm})\end{array}$ & $9,9(158)$ & $8,5-11,3$ & $10 \%(119)$ & $8,3-11-8$ & $9,7 \%(39)$ & $6,7-12,7$ & 0,92 \\
\hline $\mathrm{PA}>140 / 90(\mathrm{mmHg})$ & $9,5(152)$ & $8,1-11$ & $9,2 \%(110)$ & $7,6-10,8$ & $10,4(42)$ & $7,5-13,7$ & 0,49 \\
\hline
\end{tabular}

AFMV: Atividade Física Moderado-Vigorosa; IMC: Índice de Massa Corporal; CC: Circunferência da Cintura; DP: Desvio Padrão; PA: Pressão Arterial; IC: Intervalo de Confiança; $\mathrm{p}^{*}<0,05$ 


\section{Tabela 6}

Comparação das prevalências dos fatores de risco cardiovascular entre áreas de estudo

\begin{tabular}{|c|c|c|c|c|c|c|c|c|c|}
\hline \multirow[b]{2}{*}{ Variável } & \multicolumn{2}{|c|}{ Geral } & \multicolumn{2}{|c|}{ Exatas } & \multicolumn{2}{|c|}{ Humanas } & \multicolumn{2}{|c|}{ Biológicas } & \multirow[b]{2}{*}{ p } \\
\hline & $\%$ & IC $\quad-95 \%$ & $\% \mathrm{~N}$ & IC $-95 \%$ & $\%$ & IC $-95 \%$ & $\% \mathrm{~N}$ & IC $-95 \%$ & \\
\hline Idade $>21$ & $53,0(847)$ & $50,5-55,2$ & $57,8(376)$ & $53,8-61,7$ & $46,6(263)$ & $42,6-50,7$ & $54,0(208)$ & $49,4-59,0$ & $<0,01 *$ \\
\hline $\begin{array}{l}\text { AFMV }<150 \\
\text { min./semana }\end{array}$ & $54,6(871)$ & $51,9-57,1$ & $50,4(284)$ & $46,1-54,6$ & $61,4(399)$ & $57,5-64,9$ & 49,1 (189) & $44,2-53,8$ & $<0,01 *$ \\
\hline $\begin{array}{l}\text { Uso de tabaco } \\
\text { em } 30 \text { dias }\end{array}$ & $11,4(180)$ & $9,7-12,8$ & $11,9(67)$ & $9,3-14,6$ & $12,2(79)$ & $9,7-14,8$ & $8,9(34)$ & $6,0-12,0$ & 0,22 \\
\hline $\begin{array}{l}\text { Uso de álcool } \\
\text { em } 30 \text { dias }\end{array}$ & $64,8(1033)$ & $62,2-67,3$ & $69,5(390)$ & $65,8-73,4$ & $64,9(442)$ & $61,2-68,5$ & $57,6(221)$ & $52,6-62,2$ & $<0,01^{*}$ \\
\hline $\begin{array}{l}\text { Excesso de álcool } \\
\text { em } 30 \text { dias }\end{array}$ & $40,2(639)$ & $37,6-42,5$ & $44,6(250)$ & $40,6-48,5$ & $39,7(258)$ & $51,5-59,4$ & $34,1(131)$ & $29,4-38,8$ & $<0,01 *$ \\
\hline $\begin{array}{l}\text { Frutas }<3 \text { porções } \\
\text { no dia anterior }\end{array}$ & $92,1(1470)$ & $90,7-93,4$ & $92,5(520)$ & $90,4-94,7$ & $91,1(592)$ & $88,9-93,1$ & $93,2(358)$ & $90,4-95,6$ & 0,43 \\
\hline $\begin{array}{l}\text { Saladas/vegetais } \\
<3 \text { porções no } \\
\text { dia anterior }\end{array}$ & $80,3(1281)$ & $78,3-82,3$ & $81,3(457)$ & $78,1-84,5$ & $82,5(536)$ & $79,5-85,2$ & $75,0(288)$ & $70,3-79,2$ & $0,01 *$ \\
\hline $\begin{array}{l}\text { Consumiu salgado } \\
\text { no dia anterior }\end{array}$ & $34,7(553)$ & $32,6-37,1$ & $33,0(188)$ & $29,6-37,6$ & $37,8(246)$ & $34,3-41,5$ & $31,0(119)$ & $26,6-35,7$ & 0,06 \\
\hline $\begin{array}{l}\text { Consumiu doce no } \\
\text { dia anterior }\end{array}$ & $68,9(1099)$ & $66,8-71,2$ & $64,2(360)$ & $60,1-68,1$ & $71,1(462)$ & $67,8-74,6$ & $72,1(277)$ & $67,7-76,8$ & $<0,01 *$ \\
\hline $\mathrm{IMC} \geq 25 \mathrm{~kg} / \mathrm{m}^{2}$ & $25,9(413)$ & $23,7-\quad 28$ & $30,1(169)$ & $26,4-34$ & $23,2(151)$ & $20,5-26,6$ & $24,2(93)$ & $20,3-28,6$ & $0,02 *$ \\
\hline $\begin{array}{l}C C \geq 80 \text { feminino e } \\
\text { ? } 94 \text { masculino }(\mathrm{cm})\end{array}$ & $9,9(158)$ & $8,5-11,3$ & $10,9(61)$ & $8,4-13,4$ & $9,7(63)$ & $7,5-12$ & $8,9(34)$ & $6,0-11,7$ & 0,59 \\
\hline $\mathrm{PA}>140 / 90 \quad(\mathrm{mmHg}$ & g) $9,5(152)$ & $8,1-11$ & $9,3(52)$ & $7,0-11,8$ & $10,6(69)$ & $8,3-13,1$ & $8,1(31)$ & $5,5-10,9$ & 0,38 \\
\hline
\end{tabular}

AFMV: Atividade Física Moderado-Vigorosa; IMC: Índice de Massa Corporal; CC: Circunferência da Cintura; DP: Desvio Padrão; PA: Pressão Arterial; IC: Intervalo de Confiança; $\mathrm{p}^{*}<0,05$

\section{Discussão e Conclusões}

Os resultados demonstraram diferenças nos fatores de risco cardiovascular entre sexos, alunos calouros e formandos e entre as áreas de estudo tanto para variáveis contínuas quanto para as prevalências. O tempo gasto em AFMV e a prevalência de estudantes que não cumpriram a recomendação de 150 minutos ou mais de AFMV por semana foram superiores entre as mulheres, formandos e estudantes de cursos da área de ciências Humanas, assim como já foi observado em estudos que fizeram tais comparações. ${ }^{19,13}$
Nos EUA, o National Association of Sport and Physical Education (NASPE) em conjunto com o College and University Physical Education Concil (CUPEC) destaca a importância da prática de atividades físicas no período universitário e sugere que as instituições incorporem a disciplina de Educação Física no currículo acadêmico, como forma de aumentar a oferta desta prática aos alunos e, assim, promover o aumento do tempo em AFMV visando à saúde do estudante. ${ }^{20}$

O uso de tabaco mostra-se um importante fator de estudo nesta população. Doenças cardiovascu- 
lares já foram apresentadas como desfecho quando relacionadas com utilização de tabaco em estudos prospectivos com amostras de universitários. ${ }^{21} \mathrm{Baer}^{10}$ sugere que a personalidade e fatores emocionais são preditivos para o uso de tabaco nesta idade. Para este autor, inclusão social e auto-afirmação frente a uma nova realidade poderiam favorecer a adesão e/ou aumento deste comportamento entre os estudantes. Segundo Rigotti et al. ${ }^{25}$, o uso de tabaco, bem como o consumo de bebidas alcoólicas, poderiam estar associados ainda com a maior exposição dos estudantes à ambientes favorecedores destes tipos de comportamentos.

Neste estudo tanto a utilização de tabaco como o consumo de álcool foram observados de forma mais frequentemente em homens, formandos e alunos das ciências Exatas comparado às outras áreas, como já foi descrito anteriormente pela literatura. ${ }^{13,23,24}$

As recomendações alimentares atuais para saúde são de três ou mais porções de frutas por dia, esta mesma quantidade sugerida para o consumo de saladas e/ou vegetais, bem como a diminuição ou exclusão de alimentos ricos em carboidratos simples e com alto teor de gorduras saturadas. ${ }^{2}$ No presente estudo a prevalência de alunos que consumiram seis ou mais porções de frutas, saladas e/ou vegetais somados, no dia anterior à pesquisa foi de $13,8 \%$. Vários autores mostraram prevalências superiores, com valores que variaram de $24,9 \%$ a $35,4 \% .^{26,27,18}$ No entanto, devese levar em consideração que neste estudo foi utilizada referência proposta em 2010, de três ou mais porções de frutas e três ou mais porções de vegetais. Os publicados anteriormente a esta proposição, utilizaram recomendações sugeridas em 1988, 1997, 1999 e 2005, onde somados frutas e vegetais, a quantidade consumida deveria ser de cinco ou mais porções totais. Isto pode ter reduzido a prevalência do consumo de frutas e vegetais desta amostra, comparado às apresentadas pelos estudos citados.

Quando analisado separadamente o consumo de vegetais mostrou-se menor e menos frequente para o sexo masculino frente ao feminino e em alunos da área de ciências Humanas. Estudos similares apontaram resultados não conclusivos quanto a este comportamento. $^{28,29}$

Para verificar o consumo de alimentos nocivos à saúde cardiovascular, foram analisados consumo de salgados e doces no dia anterior à pesquisa. $\mathrm{O}$ consumo de salgados (batatas frita, chips ou similares) teve prevalência de $34,6 \%$. Este resultado se mostra infe- rior ao verificado na literatura, onde os valores variaram de $42,5 \%$ a $51,7 \% .^{30,29,31}$ Já para o consumo de doces a prevalência foi de $68,8 \%$. As proporções deste comportamento em outros estudos brasileiros também parecem altas, variando entre $77,6 \%$ e $80,6 \%$. 29,31 Thiagarajah e Torabi ${ }^{30}$ sugerem ainda que, quando se trata de alimentos salgados, parece ser mais frequente entre homens, já o consumo de doces, mais elevado em mulheres. No presente estudo, não foi observado diferença no consumo de salgado entre os sexos e o consumo de doces foi superior em mulheres.

Apesar de alguns autores sugerirem diferenças neste comportamento entre alunos calouros e formandos, não foi observado diferenças entre períodos de graduação. ${ }^{31}$ Embora escassez de estudos comparando este tipo de variável entre áreas de estudo em universitários, este comportamento diferiu dos outros, em que a área de ciências Biológicas mostrou menor prevalência de fatores de risco, neste caso o consumo de doces foi superior nesta área de estudo, comparada às ciências Humanas e Exatas. Já para o consumo de salgados não foi obtida diferença. Podese sugerir que o maior número de mulheres em cursos da área de saúde, que foram incluídos entre os cursos de ciências Biológicas, tenha elevado o consumo de doces neste estrato, visto que esta variável foi superior no sexo feminino.

A utilização de índices antropométricos é recomendada devido à praticidade e boa associação com doenças cardiovasculares e em universitários é comumente realizada desde décadas anteriores. ${ }^{7,32} \mathrm{~A}$ prevalência de alunos com IMC elevado $(25,9 \%)$ corrobora com estudos internacionais que utilizaram este indicador para avaliação de sobrepeso e obesidade em universitários. ${ }^{5,4,18} \mathrm{Na}$ comparação entre sexos, os homens mostraram maior prevalência de sobrepeso ou obesidade $(31,9 \%)$ do que as mulheres $(20 \%)$. A superioridade deste indicador no sexo masculino pode ser observada em diversos estudos, de países desenvolvidos ou em desenvolvimento. ${ }^{22,18}$ Este estudo apresentou ainda, maior prevalência de indivíduos com IMC elevado entre alunos de cursos das ciências Exatas comparado às outras áreas de estudo. Uma possível explicação pode ser o fato da maior presença de cursos em tempo integral nesta área, o que dificulta aos alunos cuidados maiores com hábitos de saúde, como atividade física e alimentação adequada. Além disso, foi verificado que alunos desta área mostraram-se frequentemente mais engajados no consumo de álcool, menor frequência em consumo de vegetais, compara- 
do aos outros cursos, além de baixa prevalência do consumo adequado de frutas.

A circunferência de cintura também é observada como importante índice preditivo de doenças cardiovasculares e sua relevância já foi descrita em estudos internacionais e nacionais. ${ }^{33,34}$ A prevalência de CC elevada neste estudo foi de $9,9 \%$, este valor parece estar bem próximo dos observados em estudos similares. ${ }^{39,35}$ Apesar de diferenças nas prevalências entre grupos de sexo, períodos de graduação e áreas de conhecimento não terem sido identificadas, os valores contínuos apontam para superioridade em homens, formandos e alunos de cursos de ciências Exatas.

O ponto de corte sugerido pela OMS4 para caracterizar a pressão arterial como elevada é de 140/ $90 \mathrm{mmHg}$ e este fator é visto como importante indicador de risco cardiovascular. ${ }^{36,36}$ A prevalência de pressão arterial elevada nos universitários deste estudo foi de $9,5 \%$. Com exceção dos dados apresentados por Simão et al. ${ }^{38}$ em estudo realizado com universitários de Lubango na Angola, onde estes encontraram alta prevalência de $20,3 \%$, outros trabalhos não apresentaram valores superiores à $11 \%$, assim como o verificado neste. ${ }^{39,22,35}$ Quando comparadas prevalências entre sexos, houve superioridade apresentada pelos homens $(14,2 \%)$ frente às mulheres $(4,9 \%)$. Esta diferença da pressão arterial entre sexos também pode ser observada em outros estudos realizados com universitários de variadas nacionalidades..$^{39,22,38,35}$ Diferente do sugerido por Buenza et al. ${ }^{40}$, onde verificaram aumento dos casos de Hipertensão em universitários, com o passar do tempo de graduação, neste estudo não foi observado diferença na prevalência de pressão arterial elevada entre calouros e formandos. Entre áreas de estudo foi verificada diferença dos valores contínuos, no entanto a prevalência de indivíduos com PA elevada não apontou diferença.

O ponto forte deste estudo foi a grande amostra representativa de alunos estratificada por sexo, períodos de estudo e áreas de conhecimento. No entanto algumas limitações podem ser apontadas. A principal delas pode ser atribuída ao método recordatório utilizado para coleta de informação sobre os comportamentos, pois, como todo questionário, deve-se contar com a integridade das informações do respondedor. No entanto, em grandes amostras, a utilização de métodos diretos é dificultada pela questão do tempo e da inviabilidade financeira. Além disso, a utilização des- tes instrumentos deve ser considerada, visto que estudos de fiabilidade e validação foram efetuados, indicando bons resultados para os questionários utilizados. ${ }^{17,41}$ Outro ponto a se ressaltar é que a estrapolação dos resultados para outras populações universitárias não é possível, visto que a amostra foi extraída de universitários da Universidade Federal do Paraná, sendo possível somente concluir acerca desta população.

As comparações da prática de AFMV entre os grupos propostos mostraram que os mais ativos foram os homens, calouros e da área de ciências Biológicas. Diante disto, mais oportunidades dentro da universidade, bem como formas de incentivar o aumento da prática de atividades físicas entre os alunos devem ser estimuladas, enfatizando ainda, os grupos menos ativos, pois menos da metade dos alunos cumpriram a recomendação desta prática.

Um em cada dez alunos reportou ter fumado nos trinta dias anteriores à pesquisa, sendo que este comportamento foi mais elevado nos homens e em estudantes formandos. O consumo de álcool teve nos trinta dias anteriores à pesquisa alta prevalência e foi superior entre homens, formandos e estudantes das ciências Exatas, assim como para o consumo excessivo de bebidas alcoólicas que foi feito por quase a metade dos alunos. Campanhas institucionais poderiam ser frequentemente elaboradas, visando à diminuição destes comportamentos deletérios à saúde dos estudantes, principalmente nos grupos mais inclinados a estes comportamentos.

Os baixos valores de consumo de frutas, saladas e/ou vegetais, e alta prevalência do consumo de doces e salgados, apontam para necessidade de um cuidado importante a ser tomado quanto ao tipo de alimentação que é disponibilizada aos estudantes nos restaurantes universitários e cantinas das instituições já que boa parte deles passa longos períodos dentro da universidade. Ao cuidar da alimentação dos estudantes, pelo menos dentro da universidade, a instituição também faz sua parte no controle do excesso de peso corporal, visto em um quarto da amostra estudada, além da pressão arterial elevada, observada em um a cada dez alunos.

Este estudo sugere ainda, como futuras pesquisas, a realização de um desenho longitudinal para acompanhamento de alunos ingressantes até a saída da universidade, com o intuito de verificar modificações nos comportamentos e fatores de risco numa mesma amostra durante este período. 


\begin{abstract}
Study design: A comparative cross-sectional study. Objective: The aim of this study is to compare risk factors among sexes, freshmen and senior students as well as fields of study. Methodology: Specific behaviors towards physical activity, smoking, alcohol consumption, dietary habits were obtained by recall method from the IPAQ and YRBSS questionnaires, measurements of weight and height for BMI, waist circumference and blood pressure were taken. Results: All continuous variables were higher for men than women $(p<0.01)$. The waist circumference and systolic blood pressure were superior in senior students than freshmen $(p<0.01)$. The waist circumference, systolic and diastolic blood pressures were higher in students from Exact Sciences compared to other fields. 54.6\% students proved insufficiently active, $64.8 \%$ consumed alcohol within the last month and $92.1 \%$ did not eat the recommended three or more servings of fruit per day. Conclusion: Important differences were observed in the risk factors related to moderate-vigorous physical activity, smoking, alcohol consumption, BMI, waist circunference and eating habits among the groups, which demonstrate the need for investments to promote changes in the students' behaviors.
\end{abstract}

Keywords: Risk Factors. Life Style. Students. Obesity. Physical Activity.

\section{Referências}

1. Hvidtfeldt UA, Tolstrup JS, Jakobsen MU, Heitmann BL, Gronbaek M, O'Reilly E, et al. Alcohol Intake and Risk of Coronary Heart Disease in Younger, MiddleAged, and Older Adults. Circulation. 2010; 121:1589-97..

2. U.S. Department of Agriculture and U.S. Department of Health and Human Services. Dietary Guidelines for Americans, 2010.

3. Lee CD, Folsom AR, Blair SN. Physical Activity and Stroke Risk: A Meta-Analysis. Stroke. 2003; 34:2475-82.

4. World Health Organization. Prevention of cardiovascular disease: Guidelines for assessment and management of total cardiovascular risk, 2007

5. Desai N, Miller WC, Staples B, Bravender T. Risk Factors Associated With Overweight and Obesity in College Students. J Am Coll Health Assoc. 2008; 57:109-14.

6. Mackey MC, Mckinney SH, Tavakoli A. Factors Related to Smoking in College Women. J of Com Health Nurs. 2008; 25: 106-21.

7. Sahi T, Paffenbarger RS, Hsieh C, Lee IM. Body Mass Index, Cigarette Smoking, and Other Characteristics as Predictors of Self-Reported, Physician-Diagnosed Gallbladder Disease in Male College Alumni. Am J Epidemiol. 1998; 147:644-51.

8. Lee I, Paffenbarger JR. Physical Activity and Stroke Incidence The Harvard Alumni Health Study. Stroke. 1998; 29: 2049-54.

9. Wood PK, Sher KJ, Rutledge PC. College student alcohol consumption, day of the week, and class schedule. Alcohol Clin Exp Res. 2007; 31: 1195-207.

10. Baer JS. Student factors: understanding individual variation in college drinking. J Stud Alcohol Suppl. 2002; 14:40-53.

11. Han JL, Dinger MK, Hull HR, Randall NB, Heesch KC, Fields DA. Changes in women's physical activity during the transition to college. Am J Health Educ. 2008; 39:194-9.

12. Racette SB, Deusinger SS, Strube MJ, Highstein GR, Deusinger $\mathrm{RH}$. Changes in weight and health behaviors from freshman through senior year of college. J Nutr Educ Behav. 2008; 40:39-42.
13. Dawnson KA, Schneider MA Fletcher PC, Bryden PJ. Examining gender differences in the health behaviors of Canadian university students. J R Soc Promot Health. 2007; 127:38-44.

14. Ribeiro M, Fernandes A. Pratice of physical activty in Young college students of public university in Bragança. Promoção da saúde e actividade fisica: Contributos para o Desenvolvimento Humano, 2010.

15. Medronho RA. Epidemiology. 2sd edition. Atheneu, São Paulo, 2009.

16. World Health Organization. Global recommendations on physical activity for health, 2010.

17. Teixeira M. YRBS-C: Traduction, transcultural adaptation and psicometrics propierties. Tesis of master degree, UEL, 2009.

18. Lowry R, Galuska DA, Fulton JE, Wechsler H, Kann L, Collins JL. Physical activity, food choice, and weight management goals and practices among U.S. college students. Am J Prev Med. 2000; 18:18-27.

19. Fontes ACD, Vianna RPT. Prevalence and associated factors with the low level of physical activity among college students of a public university in Nordest. Brazil. Rev Bras Epidemiol. 2009; 12: 20-9.

20. National Association of Sport and Physical Education (NASPE) and College and University Physical Education Concil (CUPEC). College/University Physical Activity Instruction Programs: A Critical Piece in the Education of Young Adults: A Position Paper from the National Association for Sport and Physical Education, 2007.

21. Johnson HM, Gosset LK, Piper ME, Aeschlimann SE, Korcarz $\mathrm{CE}$, Baker TB, et al. Effects of smoking and smoking cessation on endothelial function: 1-Year: Outcomes from a randomized clinical trial. J Am Coll Cardiol. 2010; 55:1-8.

22. Oviedo G, Morón de Salin A, Santos I, Sequera S, Soufrontt G, Suárez $\mathrm{P}$, et al. Factores de riesgo de enfermedades crónicas no transmisibles en estudiantes de la carrera de medicina. Universidad de Carabobo, Venezuela. Año 2006. Nutr Hosp. 2008; 23:288-93.

23. Vankim NA, Laska MN, Ehlinger E, Lust K, Story M. Understanding young adult physical activity, alcohol and tobacco use in community colleges and 4-year post-secondary institutions: A crosssectional analysis of epidemiological surveillance data. BMC Public Health. 2010; 10: 208. 
24. Simão CB, Nahas MV, Oliveira ESA. Physical activity, eating habits and prevalence of overwheigt and obesity among college students of plateau catarinense university. uniplac, lages. S.C . Rev Bras Ativ Fís Saúde. 2006; 11:3-12.

25. Rigotti NA, Moran SE, Wechsler H. US College Students' Exposure to Tobacco Promotions: Prevalence and Association with Tobacco Use. Am J Public Health. 2005; 95: 138-44.

26. Laska MN, Pasch KE, Lust K, Story M, Ehlinger E. Latent class analysis of lifestyle characteristics and health risk behaviors among college youth. Prev Sci. 2009; 10:376-86.

27. Ko M. The comparison in daily intake of nutrients, dietary habits and body composition of female college students by body mass índex. Nut Res Pract. 2007; 2: 131-42.

28. Feitosa EPS, Dantas CAO, Andrade-Wartha ERS, Marcellini PS, Mendes-Netto RS. Eating behaviors of college students in a Nordest public university, Brazil. Aliment Nutr. 2010; 21 : 225-30.

29. Colares V, Franca C, Gonzalez E. Health Behaviors among colleges: diferences between genres. Cad Saúde Pública. 2009; 25: 521-8.

30. Thiagarajah K, Torabi M. Irregular Breakfat Eating and Associated Health Beahaviors: A Pilot Study Among College Students. Health Educ. 2009;41: 4-10.

31. Franca C, Colares V. Comparative study of health behaviors between freshman and seniors college students. Rev Saúde Pública. 2008; 42:420-7.

32. Paffenbarger RS, Hyde RT, Wing AL, Lee IM, Jung DL, Kampert JB. The association of changes in physical-activity level and other lifestyle characteristics with mortality among Men. N Engl J Med. 1993; 328: 538-45.

33. Sarno F, Monteiro CA. The relative importance of body mass índex and the waist circunference to predict the hypertension. Rev Saúde Pública. 2007; 41: 788-96.
34. Janssen I, Katzmarzyk P, Ross R. Body Mass Index, Waist Circumference, and Health Risk. Arch Intern Med. 2002; 162: 2024-79.

35. Deschamps EG, Exsome CP, Sánchez JV, Flores BT, Bastida $\mathrm{S}$, Vaquero MP, et al. Prevalencia de síndrome metabólico y sua sociación con el índice de masa corporal en universitários. Med Clin. 2007; 129:766-9.

36. Cook NR, Obarzanek E, Cutler JA, Buring JE, Rexrode KM, Kumanyika SK, et al. Joint effects of sodium and potassium intake on subsequent cardiovascular disease: the Trials of Hypertension Prevention follow-up study. Arch Intern Med. 2009; 169: 32-40.

37. Giles TD, Materson BJ, Cohn JN, Kostis JB. Definition and classification of hypertension: an update. J Clin Hypert. 2009; 11: 611-4.

38. Simão M, Hayashida M, Santos CB, Cesarino EJ, Nogueira MS. Arterial hypertension among college students of Lubango city, Angola. Rev Latino-am Enfermagem 2008;16: 672-8.

39. Martins MCC, Ricarte IF, Rocha CHL, Maia RB, Silva VB, Veras $A B$, et al. Blood pressure, excess weight and level of physical activity in students of a public university. Arq Bras Cardiol. 2010; 95:192-9.

40. Buenza JJ, Martínez-Gonzáles MA, Serrano-Martínez M, Alonso A. Incidência de hipertensión en una cohorte de graduados universtarios españoles: el studio SUN. Rev Esp Cardiol. 2006; 59: 1331-4.

41- Pardini R. Matsudo SM, Araújo T, Matsudo V, Andrade E, Braggion G, et al. Validation of International Physical Activity Questionaire (IPAQ-6). Pilot study with brazilians youngs adults. Rev Bras Ciênc Mov. 2001; 9: 45-51. 